Tropical Journal of Pharmaceutical Research December 2020; 19 (12): 2521-2527

ISSN: $1596-5996$ (print); 1596-9827 (electronic)

(C) Pharmacotherapy Group, Faculty of Pharmacy, University of Benin, Benin City, 300001 Nigeria

Original Research Article

http://dx.doi.org/10.4314/tjpr.v19i12.6

\title{
CD147 promotes melanoma cell growth via SOX4-mediated glycolytic metabolism
}

\author{
Xiaohui Sun', Pengfei Yang ${ }^{2}$, Yuan Jiang ${ }^{3 *}$ \\ ${ }^{1}$ Department of Dermatology, Shandong Provincial Qianfoshan Hospital, Cheeloo College of Medicine, Shandong University, \\ Jinan, Shandong Province 250014, '2Department of Dermatology, The First Affiliated Hospital of Shandong First Medical \\ University \& Shandong Provincial Qianfoshan Hospital, Jinan, Shandong Province 250014, ${ }^{3}$ Department of Plastic Burn and \\ Wound Repair, Lishui People's Hospital, Lishui, Zhejiang Province 323000, China
}

*For correspondence: Email: jiangyuan0818@163.com; Tel: +86-0578-2780253

Sent for review: 9 September 2020

Revised accepted: 23 November 2020

\begin{abstract}
Purpose: To determine the functional roles of cluster of differentiation 147 (CD147) in glycolysis in melanoma cells.

Methods: CD147 expression in melanoma tissue and adjacent normal tissue was determined using quantitative real time polymrase chain reaction (qRT-PCR) and immunohistochemistry. Cell Counting Kit-8 (CCK-8) and colony formation assays were used to evaluate cell viability and colony formation, respectively. The role of CD147 in glycolysis in melanoma cells was investigated by determining glucose uptake, production of lactate, and cellular level of ATP.

Results: CD147 was enhanced more in melanoma tissue than that in the adjacent normal tissue $(p<$ 0.001). CD147 overexpression promoted the viability and colony formation of melanoma cells. On the other hand, CD147 silencing decreased the viability and colony formation of melanoma cells. Glucose uptake, production of lactate, and cellular level of ATP were upregulated in melanoma cells by CD147 overexpression and downregulated by shRNA-mediated depletion of CD147. CD147 increased expression of $\mathrm{C}-\mathrm{X}-\mathrm{C}$ motif chemokine ligand 1 (CXCL1) to activate the sex-determining region Y-related high-mobility group box 4 (SOX4) pathway. Knockdown of CXCL1 attenuated the positive regulatory effect of CD147 on SOX4. Besides, overexpression of SOX4 reversed the suppressive effects of CD147 silencing on melanoma cell viability, colony formation, and glycolysis.

Conclusion: CD147 contributes to melanoma cell growth via upregulation of SOX-mediated glycolysis, thus providing a therapeutic avenue for the management of melanoma.
\end{abstract}

Keywords: Cluster of differentiation 147, CD147, Sex-determining region Y-related high-mobility group box 4, Melanoma, Cell growth, Glycolysis

\footnotetext{
This is an Open Access article that uses a funding model which does not charge readers or their institutions for access and distributed under the terms of the Creative Commons Attribution License (http://creativecommons.org/licenses/by/4.0) and the Budapest Open Access Initiative (http://www.budapestopenaccessinitiative.org/read), which permit unrestricted use, distribution, and reproduction in any medium, provided the original work is properly credited.
}

Tropical Journal of Pharmaceutical Research is indexed by Science Citation Index (SciSearch), Scopus, International Pharmaceutical Abstract, Chemical Abstracts, Embase, Index Copernicus, EBSCO, African Index Medicus, JournalSeek, Journal Citation Reports/Science Edition, Directory of Open Access Journals (DOAJ), African Journal Online, Bioline International, Open-J-Gate and Pharmacy Abstracts

\section{INTRODUCTION}

Melanoma is considered as the most aggressive type of skin cancer, and patients with local or distant metastasis of melanoma have a poor prognosis [1]. The treatment for melanoma remains a challenge due to pre-existing or acquired drug resistance [2]. It is thus indispensable to find new and alternative therapeutic factors for melanoma. 
Cluster of differentiation 147 (CD147), a transmembrane glycoprotein widely expressed in tissue and cells, facilitates secretion of matrix metalloproteinases to promote tumor cell metastasis [3]. In melanoma cells, CD147 induced secretion of matrix metalloproteinases and promoted cell invasion [4]. Moreover, CD147 promoted tumor angiogenesis in melanoma through upregulation of vascular endothelial growth factor receptor 2 [5], and promoted anaerobic glycolysis in melanoma cells through interaction with monocarboxylate transporters [6]. However, little report considering the downstream target involved in CD147-mediated aerobic glycolysis in melanoma cells.

Sex-determining region Y-related high-mobility group box 4 (SOX4) is a transcription factor that can regulate embryonic development and cell differentiation, promote or suppress malignant cell progression [7]. In melanoma cells, SOX4 could suppress cellular apoptosis through binding to p65 promoter [8], and stimulate melanoma cell proliferation through promotion of glycolysis [9]. This study hypothesized that SOX4 was implicated in CD147-mediated glycolysis of melanoma cells.

This study was performed to clarify the functional roles of CD147 in glycolysis and proliferation of melanoma cells, and then identify the role of the CD147 was depending on regulating SOX4, providing a therapeutic factor for melanoma treatment.

\section{EXPERIMENTAL}

\section{Preparation of tumor tissue}

78 patients diagnosed with melanoma were collected from our hospital. Melanoma tissue biopsy was obtained from these patients. Tissues were then used for quantitative PCR (qPCR) and immunohistochemical analysis. This study was approved by the Ethics Committee of our hospital (Approval no.2018015), and written informed consent was obtained. All procedures were performed in accordance with the 1964 Helsinki Declaration and its later amendments [10].

\section{Immunohistochemistry}

Formalin-fixed and paraffin-embedded melanoma tumor tissue and adjacent non-tumor tissue were cut into sections in 4- $\mu \mathrm{m}$. Following antigen retrieval, the sections were blocked with $3 \% \mathrm{H}_{2} \mathrm{O}_{2}$ and incubated with primary antibody against CD147 (1:500; Abcam, Cambridge, UK), followed by incubated with biotinylated secondary antibody (1:500; Abcam). Finally, the sections were counterstained with hematoxylin before examination by a light microscope (Olympus, Tokyo, Japan).

\section{Cell culture}

Normal human epidermal melanocytes (HEMaLP) and melanoma cell lines (SK-MEL-2, SKMEL-1, SK-MEL-28, A375) were purchased from Chinese Academy of Sciences (Shanghai, China). The cells were cultured in Dulbecco's Modified Eagle Medium (DMEM; Lonza, Basel, Switzerland) containing $10 \%$ fetal bovine serum (FBS, Lonza) in a $37^{\circ} \mathrm{C}$ incubator.

\section{Plasmid construction and cell transfection}

The sequences for full-length CD147, CXCL1, and SOX4 were inserted into pcDNA3.1 vector (Invitrogen, Carlsbad, CA, USA). Short hairpin RNAs (shRNAs) targeting CD147 and CXCL1 were synthesized by GenePharma (Suzhou, China). For transfecting pcDNA3.1 vectors or shRNAs A into 375 or SK-MEL-2 cells, Lipofectamine 2000 (Invitrogen) was used.

\section{Cell viability and colony formation assay}

For cell viability assay, A375 or SK-MEL-2 cells were seeded $\left(2 \times 10^{4} /\right.$ well $)$ into 96 -well plates and cultured for various duration times $(0,24,48$, and $72 \mathrm{~h})$. Cells were treated with CCK 8 solution (Dojindo, Tokyo, Japan) for $4 \mathrm{~h}$. Absorbance at $450 \mathrm{~nm}$ was measured. For colony formation assay, A375 or SK-MEL-2 cells were seeded (2 $\times 10^{2}$ /well) into 6-well plates, and then cultured in DMEM, with the medium replaced with fresh medium every 3 days. Colonies were fixed and stained, and then examined under a microscope (Olympus) two weeks later.

\section{Determination of glucose uptake, production of lactate, and cellular level of ATP}

Transfected cells (A375 or SK-MEL-2) were cultured in DMEM. Forty-eight hours later, glucose uptake, production of lactate, and cellular level of ATP in each well were evaluated using Glucose Uptake-Glo Assay (Promega, Madison, WI, USA); Lactate Assay Kit (BioVision, Mountain View, CA, USA); and CellTiterGlo® Luminescent Cell Viability Assay (Promega), respectively.

\section{Quantitative polymerase chain reaction (qPCR)}

The extraction of total RNA from paired melanoma tissue and adjacent non-tumor tissue 
were achieved by TRIzol reagent (Invitrogen). The reverse transcription of cDNAs was synthesized by the PrimeScript ${ }^{\mathrm{TM}}$ RT Reagent Kit (TaKaRa, Tokyo, Japan), and the amplification was achieved by qPCR using SYBR Premix Ex Taq II Kit (TaKaRa). Glyceraldehyde-3phosphate dehydrogenase (GAPDH) was used as internal control with the listed primer sequences (Table 1).

Table 1: Primer sequences

\begin{tabular}{lll}
\hline & Forward & Reverse \\
\hline CD1 & 5'- & 5'- \\
$\mathbf{4 7}$ & AGGACCGGCGAGG & TGCAAGCACTGGG \\
& AATAGGA-3' & AGTGGAC-3' \\
GAP & 5'- & 5'- \\
DH & GCAATGCCTCCTG & CCCCAGCGTCAAA \\
& CACCACCA-3' & GGTGGAGG-3' \\
\hline
\end{tabular}

\section{Western blotting}

Harvested A375 or SK-MEL-2 cells were lysed and the protein concentration was firstly determined. Proteins $(30 \mu \mathrm{g})$ were separated by sodium dodecyl sulfate-polyacrylamide electrophoresis (SDS-PAGE) and electrotransferred to PVDF membranes. Following blocking with $5 \%$ bovine serum albumin in phosphate-buffered saline (PBS) for $1 \mathrm{~h}$, membranes were incubated overnight with specific primary antibodies against CD147 (1:1500; Abcam), CXCL1 (1:1500; Abcam), SOX4 (1:2000; Abcam), hexokinase 2 (HK2) (1:2000; Abcam), lactate dehydrogenase A (LDHA) (1:2500; Abcam) and GAPDH (1:3500; Abcam). After incubation with the horseradish peroxidase-labeled secondary antibody (1:5000; Abcam), specific protein bands in each membrane were examined.

\section{Statistical analysis}

Each experiment was repeated at least three times, and results were shown as mean \pm standard deviation (SD). By using GraphPad Prism 6.00 (GraphPad Software, San Diego, CA, USA), statistical analysis was carried out using Student's $t$-test or one-way analysis of variance (ANOVA). $P<0.05$ was considered statistically significant.

\section{RESULTS}

\section{Enhanced expression of CD147 in melanoma}

Results showed that CD147 was enhanced in melanoma tissue compared to non-tumor tissue $(p<0.001)$ (Figure 1 A and B). Significant upregulation of CD147 was also observed in melanoma cells (SK-MEL-2, SK-MEL-1, SK-
MEL-28, and A375) compared to HEMa-LP normal human melanocytes $(p<0.001$; Figure 1 C), suggesting the participation of CD147 in melanoma progression.

$A$

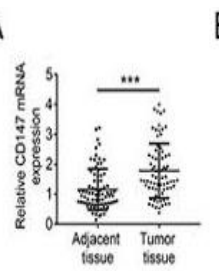

B

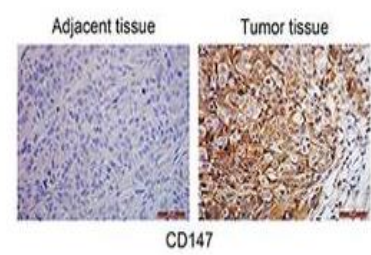

C
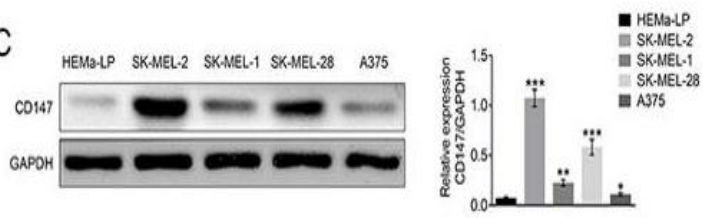

Figure 1: Enhanced expression of CD147 in melanoma. Upregulation of CD147 in melanoma tissue compared to adjacent non-tumor tissue determined by GPCR (A) and immunohistochemical (B) analysis. (C) Upregulation of CD147 in melanoma cells compared to HEMa-LP normal human melanocytes determined by western analysis (left) and qPCR analysis (right). ${ }^{*} P<0.05,{ }^{* *} p<0.01,{ }^{* * *} p<$ 0.001

\section{CD147 contributed to melanoma cell growth}

Significant upregulation of CD147 was verified in A375 cells transfected with pcDNA-CD147 compared to that with empty vector $(p<0.001)$ (Figure $2 \mathrm{~A}$ ). A significant downregulation of CD147 was verified in SK-MEL-2 cells transfected with shCD147 \#1 or \#2 compared to that transfected with negative control shRNA $(\operatorname{shNC})(p<0.001$, Figure 2 A). Melanoma cell viability (Figure $2 \mathrm{~B}$ ) and colony formation (Figure 2 C) were enhanced by CD147 overexpression, and reduced by shRNA-mediated CD147 silencing. These results revealed that CD147 contributed to melanoma cell growth.

\section{CD147 promoted glycolysis in melanoma cells}

Results indicated a significant increase in glucose uptake (Figure $3 \mathrm{~A}$ ), production of lactate (Figure $3 \mathrm{~B}$ ), and cellular level of ATP (Figure $3 C)$ in A375 cells, which caused by CD147 overexpression $(p<0.01)$. However, shRNAmediated silencing of CD147 in SK-MEL-2 cells decreased glucose uptake (Figure $3 \mathrm{~A}$ ), production of lactate (Figure $3 \mathrm{~B}$ ), and cellular level of ATP (Figure $3 \mathrm{C}$ ), suggesting that CD147 promoted glycolysis in melanoma cells.

Trop J Pharm Res, December 2020; 19(12): 2523 
A

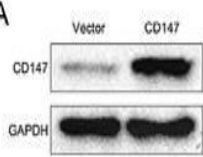

A375
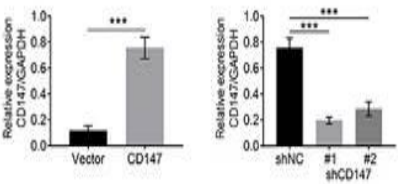

C
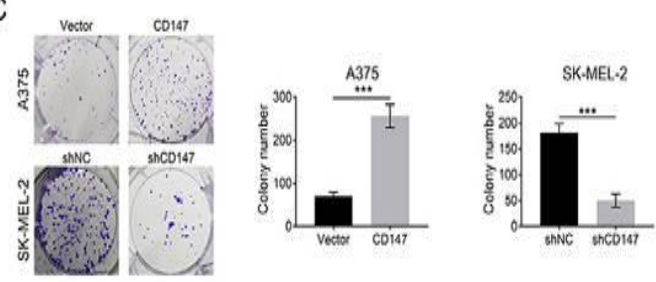

Figure 2: CD147 contributed to melanoma cell growth. Upregulation of CD147 in A375 cells transfected with pcDNA-CD147 (A), increased cell viability (B) and colony formation (C). Downregulation of CD147 in SKMEL-2 cells transfected with shCD147 \#1 OR \#2 (A), decreased cell viability (B) and colony formation (C). ${ }^{* \star} P<0.01,{ }^{* * *} p<0.001$

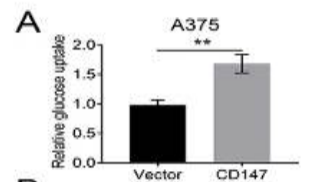

B
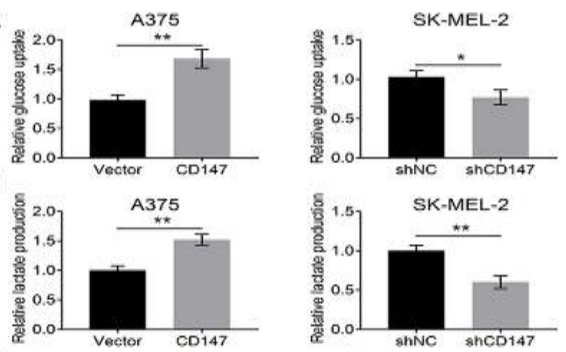

C
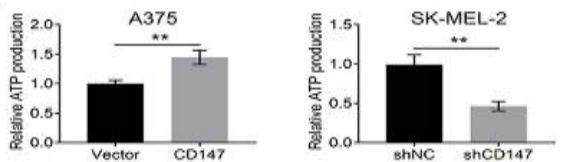

Figure 3: CD147 promoted glycolysis in melanoma cells. Transfection with pcDNA-CD147 increased glucose uptake (A), production of lactate (B), and ATP (C) by A375 cells. Transfection with shCD147 decreased glucose uptake (A), production of lactate (B), and ATP (C) of SK-MEL-2 cells. ${ }^{*} P<0.05,{ }^{* *} p<$ 0.01

\section{CD147 positively regulated SOX4 via CXCL1}

CD147 overexpression in A375 cells enhanced CXCL1 and SOX4 expression (Figure $4 \mathrm{~A}$ ), suggesting the promotive effect of CD147 on CXCL1 and SOX4 expression in melanoma cells. Moreover, knockdown of CXCL1 attenuated the promotive effect of CD147 on CXCL1 and SOX4 expression in A357 cells (Figure 4 B) Overexpression of CXCL1 reversed the CD147 silencing-induced decrease in CXCL1 and SOX4 (Figure $4 \mathrm{~B}$ ), suggesting that CD147 upregulates SOX4 in melanoma cells through CXCL1.

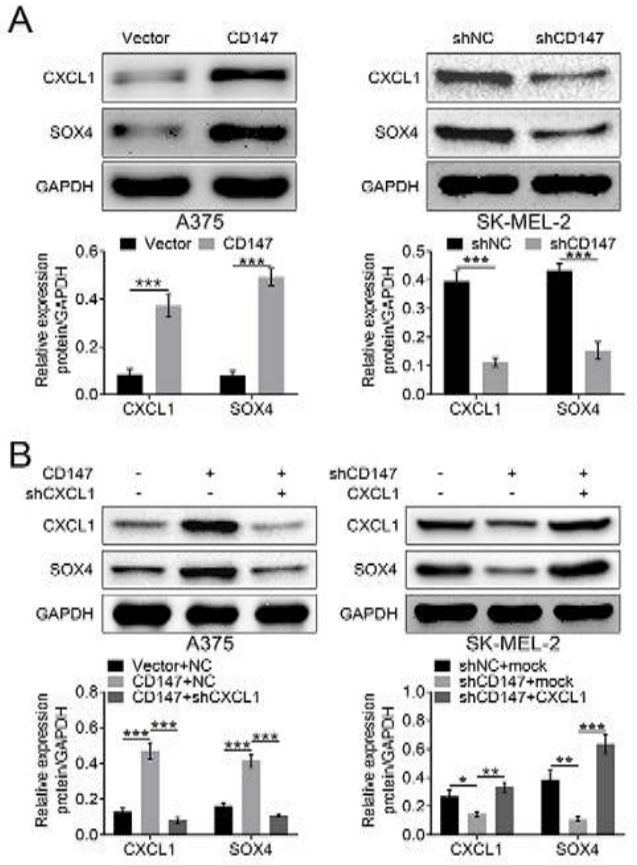

Figure 4: CD147 positively regulated SOX4 through CXCL1. Transfection with pcDNA-CD147 upregulated CXCL1 and SOX4 protein expression in A375 cells, and transfection with shCD147 decreased CXCL1 and SOX4 expression in SK-MEL-2 cells (A). Knockdown of CXCL1 attenuated the promotive effect of CD147 on CXCL1 and SOX4 expression in A357 cells, whereas CXCL1 overexpression increased CD147 silencing-induced decrease in CXCL1 and SOX4 (B). ${ }^{\star} P<0.05,{ }^{* *} p<0.01,{ }^{* * *} p<0.001$

\section{CD147 promoted glycolysis in melanoma cells via upregulation of SOX4}

Overexpression of SOX4 reversed CD147 knockdown-induced decrease in cell viability in SK-MEL-2 cells (Figure $5 \mathrm{~A}$ ), and counteracted the suppressive effects of CD147 knockdown on glucose uptake (Figure $5 \mathrm{~B}$ ), production of lactate (Figure $5 \mathrm{C}$ ), and cellular level of ATP (Figure 5 D) in SK-MEL-2 cells. Furthermore, knockdown of CD147 downregulated enzymes involved in aerobic glycolysis, including HK2 and LDHA (Figure $5 \mathrm{E}$ ). However, SOX4 overexpression promoted CD147 knockdown-induced decrease in hexokinase 2 (HK2) and lactate dehydrogenase $A$ (LDHA) (Figure 5 E), suggesting that CD147 promoted glycolysis in melanoma cells through upregulation of SOX4.

Trop J Pharm Res, December 2020; 19(12): 2524 
A
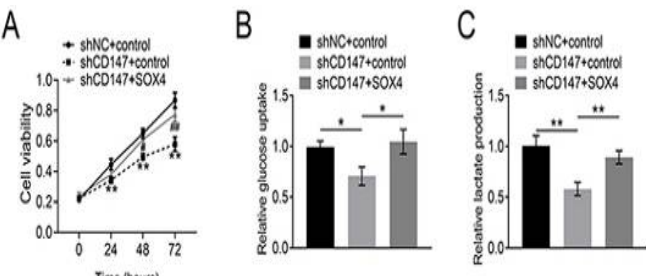

D

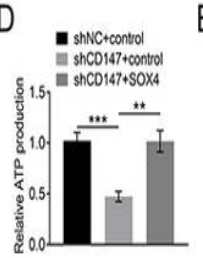

E
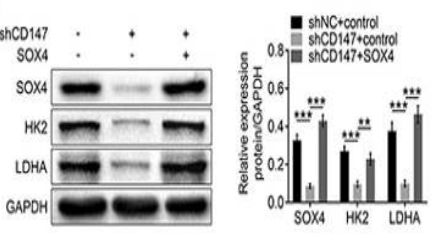

Figure 5: CD147 contributed to glycolysis in melanoma cells through upregulation of SOX4. Overexpression of SOX4 reversed CD147 knockdowninduced decrease in cell viability (A), glucose uptake (B), production of lactate (C), cellular level of ATP (D) and expressions of HK2 and LDHA (E) in SK-MEL-2 cells. ${ }^{*} P<0.05,{ }^{* *} p<0.01,{ }^{* * *} p<0.001$

\section{DISCUSSION}

Tumor cells, with unrestrained growth ability, take up glucose and produce lactate through activation of aerobic glycolysis [11]. Manipulation of aerobic glycolysis has been regarded as a therapeutic strategy for melanoma treatment [12]. Silencing of CD147 has been reported to abrogate the expression of monocarboxylate transporters and decrease production of ATP and glycolysis in melanoma cells [6]. The mechanism involved in CD147-mediated melanoma glycolysis was investigated in this study.

Immunohistochemical analysis of CD147 expression in melanoma tissue showed that CD147 was overexpressed in melanoma, and high CD147 expression predicted poor overall survival in patients with melanoma, suggesting its prognostic role in melanoma [13]. Upregulation of CD147 in melanoma tissue and cells was also verified in this study. Zhang et al have shown that CD147 contributes to melanoma cell growth [14]. Similarly, results from CCK8 and colony formation assays in our study confirmed the promotive effect of CD147 on melanoma cell growth.

Moreover, antibody against CD147 has been reported to dissociate the CD147monocarboxylate transporter complex, thereby repressing glucose uptake, as well as lactate and ATP production in melanoma cells [15]. The results of this study also demonstrated that CD147 promoted glycolysis in melanoma cells through promotion of glucose uptake, and production of lactate and ATP. Glycolytic enzymes, including HK2 [16] and LDHA [17], were downregulated by CD147 knockdown in melanoma cells. These investigations showed that CD147 promoted melanoma cell viability, colony formation and glycolysis.

CXCL1 has been shown to increase expression of glycolytic enzymes HK2 and LDHA and promote glycolysis [18], contributing to melanoma tumorigenesis [19]. CD147 could increase CXCL1 expression through the PI3K/AKT pathway [20]. The present study revealed that overexpression of CD147 promoted CXCL1 expression in melanoma cells, whereas CD147 knockdown decreased CXCL1 expression. Moreover, CXCL1 has been shown bind to the promoter region of SOX4 and activate SOX4 transcription [21]. Silencing of CXCL1 attenuated the promotive effect of CD147 on SOX4 expression in melanoma cells, suggesting the possible regulatory ability of the CD147/CXCL1/SOX4 axis in melanoma cell growth and glycolysis.

Further functional assays revealed that overexpression of SOX4 counteracted the suppressive effects of CD147 knockdown on melanoma cell viability, glucose uptake, and production of lactate and ATP, indicating that CD147 promoted melanoma cell growth and glycolysis through upregulation of SOX4. Inactivation of the AKT pathway was implicated in SOX4-mediated increase in HK2, LDHA, and glucose transporter type 1 during glycolytic metabolism in melanoma cells [9]. Results of this study demonstrated that SOX4 overexpression reversed CD147 silencing-induced decrease in HK2 and LDHA expressions in melanoma cells. However, SOX4 was also found to suppress melanoma cell invasion [22], and knockdown of SOX4 enhanced melanoma cell migration and invasion through upregulation of $p 50$ [23]. The pathways involved in CD147/SOX4-mediated melanoma cell growth and glycolysis need to be further investigated.

\section{CONCLUSION}

This study has demonstrated that CD147 is a vital mediator of cell growth and glucose metabolism in melanoma cells. SOX4 is associated with CD147-mediated melanoma cell growth and glycolysis. The regulatory role of the newly identified CD147/SOX4 axis in melanoma glucose metabolism and cell growth provides a potential therapeutic target for the treatment of melanoma. 


\section{DECLARATIONS}

\section{Conflict of interest}

No conflict of interest is associated with this work.

\section{Contribution of authors}

We declare that this work was done by the authors named in this article and all liabilities pertaining to claims relating to the content of this article will be borne by the authors. Xiaohui Sun and Pengfei Yang designed the study, supervised the data collection, analyzed the data, and interpreted the data. Yuan Jiang prepared the manuscript for publication and reviewed the draft of the manuscript. All authors have read and approved the manuscript. Xiaohui Sun and Pengfei Yang contributed equally to the work.

\section{Open Access}

This is an Open Access article that uses a funding model which does not charge readers or their institutions for access and distributed under the terms of the Creative Commons Attribution License (http://creativecommons.org/licenses/by/ 4.0) and the Budapest Open Access Initiative (http://www.budapestopenaccessinitiative.org/rea d), which permit unrestricted use, distribution, and reproduction in any medium, provided the original work is properly credited.

\section{REFERENCES}

1. Fraihat A, Alatrash L, Abbasi R, Abu-Irmaileh B, Hamed S, Mohammad M, Abu-Rish E, Bustanji Y. Inhibitory effects of methanol extracts of selected plants on proliferation of two human melanoma cell lines. Trop $J$ Pharm Res 2018; 17(8): 1645.

2. Tentori L, Lacal PM, Graziani G. Challenging resistance mechanisms to therapies for metastatic melanoma. Trends Pharmacol Sci 2013; 34(12): 656-666.

3. Landras A, Reger de Moura C, Jouenne F, Lebbe C, Menashi S, Mourah S. CD147 Is a Promising Target of Tumor Progression and a Prognostic Biomarker. Cancers 2019; 11(11): 1803.

4. Kanekura T, Chen X, Kanzaki T. Basigin (CD147) is expressed on melanoma cells and induces tumor cell invasion by stimulating production of matrix metalloproteinases by fibroblasts. Int J Cancer 2002; 99(4): 520-528.

5. Bougatef F, Menashi S, Khayati F, Naïmi B, Porcher R, Podgorniak M-P, Millot G, Janin A, Calvo F, Lebbé $C$ et al. EMMPRIN promotes melanoma cells malignant properties through a HIF-2alpha mediated upregulation of VEGF-receptor-2. PloS one 2010; 5(8): e12265e12265.

6. Kanekura $T$, Chen $X$. CD147/basigin promotes progression of malignant melanoma and other cancers. J Dermatol Sci 2010; 57(3): 149-154.

7. Hanieh $H$, Ahmed EA, Vishnubalaji R, Alajez NM. SOX4: Epigenetic regulation and role in tumorigenesis. Semin Cancer Biol 2019.

8. Cheng Q, Du J, Xie L, Liu X, Li Z, Zuo F, Wu J, Xu J. Inhibition of SOX4 induces melanoma cell apoptosis via downregulation of NF-kappaB p65 signaling. Oncol Rep 2018; 40(1): 369-376.

9. Dai W, XuX, Li S, Ma J, Shi Q, Guo S, Liu L, Guo W, Xu $P, \mathrm{He} Y$ et al. SOX4 Promotes Proliferative Signals by Regulating Glycolysis through AKT Activation in Melanoma Cells. J Invest Dermatol 2017; 137(11): 2407-2416.

10. Association WM. World Medical Association Declaration of Helsinki. Ethical principles for medical research involving human subjects. Bull WHO 2001; 79(4): 373.

11. Updegraff BL, Zhou X, Guo Y, Padanad MS, Chen $P-H$, Yang $C$, Sudderth J, Rodriguez-Tirado C, Girard L, Minna JD et al. Transmembrane Protease TMPRSS11B Promotes Lung Cancer Growth by Enhancing Lactate Export and Glycolytic Metabolism. Cell reports 2018; 25(8): 2223-2233.e2226.

12. Mazar J, Qi F, Lee B, Marchica J, Govindarajan S, Shelley J, Li J-L, Ray A, Perera RJ. MicroRNA 211 Functions as a Metabolic Switch in Human Melanoma Cells. Molecular Cellular Biol 2016; 36(7): 1090-1108.

13. Caudron A, Battistella M, Feugeas JP, Pages C, BassetSeguin N, Mazouz Dorval S, Funck Brentano E, Sadoux A, Podgorniak MP, Menashi S, et al. EMMPRIN/CD147 is an independent prognostic biomarker in cutaneous melanoma. Exp Dermatol 2016; 25(8): 618-622.

14. Zhang $X$, Huang $Z$, Guo $Y$, Xiao T, Tang L, Zhao S, Wu $L$, Su J, Zeng $W$, Huang $H$ et al. The phosphorylation of CD147 by Fyn plays a critical role for melanoma cells growth and metastasis. Oncogene 2020; 39(21): 41834197.

15. Baba M, Inoue $M$, Itoh K, Nishizawa Y. Blocking CD147 induces cell death in cancer cells through impairment of glycolytic energy metabolism. Biochem Biophys Res Commun 2008; 374(1): 111-116.

16. Lu J, Liang $X$, Gao $Y, F u$, Shen Q. Hexokinase2 controls angiogenesis in melanoma by promoting aerobic glycolysis and activating the p38-MAPK signaling. J Cell Biochem 2019; 120(12): 19721-19729.

17. Ždralević $M$, Brand A, Di lanni L, Dettmer K, Reinders J, Singer K, Peter K, Schnell A, Bruss C, Decking S-M et al. Double genetic disruption of lactate dehydrogenases $A$ and $B$ is required to ablate the "Warburg effect" restricting tumor growth to oxidative metabolism. The $J$ Biol Chem 2018; 293(41): 15947-15961.

18. Zhuo C, Wu X, Li J, Hu D, Jian J, Chen C, Zheng $X$, Yang $C$. Chemokine ( $C-X-C$ motif) ligand 1 is associated with tumor progression and poor prognosis in patients

Trop J Pharm Res, December 2020; 19(12): 2526 
with colorectal cancer. Bioscience reports 2018; 38(4): BSR20180580.

19. Dhawan $P$, Richmond A. Role of CXCL1 in tumorigenesis of melanoma. J Leukocyte Biol 2002; 72(1): 9-18.

20. Shi $W-P$, Ju $D$, Li H, Yuan L, Cui J, Luo D, Chen Z-N, Bian H. CD147 Promotes CXCL1 Expression and Modulates Liver Fibrogenesis. Int J Molecular Sci 2018; 19(4): 1145

21. Wang $N$, Liu W, Zheng Y, Wang S, Yang B, Li M, Song J, Zhang $F$, Zhang $X$, Wang $Q$ et al. CXCL1 derived from tumor-associated macrophages promotes breast cancer metastasis via activating NF-KB/SOX4 signaling. Cell Death Dis 2018; 9(9): 880-880.

22. Jafarnejad SM, Ardekani GS, Ghaffari M, Martinka M, Li G. Sox4-mediated Dicer expression is critical for suppression of melanoma cell invasion. Oncogene 2013; 32(17): 2131-2139.

23. Jafarnejad SM, Wani AA, Martinka M, Li G. Prognostic significance of Sox 4 expression in human cutaneous melanoma and its role in cell migration and invasion. The Amer J Pathol 2010; 177(6): 2741-2752. 